\title{
Staatsfinanzen in der Eurozone: Ansätze zur Bewältigung der aktuellen Herausforderungen
}

\author{
von Wolfgang Schäuble
}

Mehr als vier Jahre nach ihrem Ausbruch ist die Finanzkrise noch lange nicht beendet. Die sogenannte Subprime-Krise, die in den USA ihren Ausgang nahm, hatte sich rasch zu einer weltweiten Finanz- und Wirtschaftskrise ausgeweitet. Inzwischen aber haben wir es mit einer Staatsschuldenkrise in einer Reihe von souveränen Staaten der Eurozone zu tun, die in der Folge dramatisch steigender Refinanzierungskosten stark unter den Druck der Märkte geraten sind. Ursächlich ist jedoch in erster Linie nicht die Finanzkrise, sondern die seit teilweise vielen Jahren nicht mehr solide Haushaltslage dieser Länder, deren Situation dann im Zuge der Krise zusätzlich noch durch Marktübertreibungen verschärft worden ist.

Vor dem Hintergrund erheblicher Verschuldung sowohl im privaten als auch im öffentlichen Sektor bedarf es in den Industriestaaten nun einer Phase der „Bilanzbereinigungen“. Der Abbau des Schuldenüberhangs und der Fremdfinanzierung im Privatsektor sowie im Finanzsystem ist zu einer Grundvoraussetzung für nachhaltiges Wachstum geworden. Die Krisen der letzten Jahre lehren uns zudem, dass die Märkte die Schuldenstände im öffentlichen Sektor nicht mehr wie bisher tolerieren, sondern sich des damit verbundenen Risikos bewusst geworden sind und entsprechend höhere Renditen verlangen.

Wir werden die Überschuldungsprobleme in den betroffenen Ländern nicht dadurch in den Griff bekommen, dass Regierungen und Zentralbanken über das bisherige Niveau hinaus weitere Mittel und Garantien zur Verfügung stellen. Es ist vielmehr von entscheidender Bedeutung, dass sich die G 20-Länder an ihre Verpflichtung halten, bis zum Jahr 2013 ihre Defizite erheblich zu reduzieren und bis zur Mitte des Jahrzehnts ihre Schuldenstände zu stabilisieren. Wir sind spätestens jetzt an dem Punkt angelangt, an dem wir unsere Konsolidierungsanstrengungen nicht mehr weiter in die Zukunft verschieben können. Dasselbe gilt für die Bekämpfung systemischer Risiken im Finanzsektor durch bessere Aufsicht und Regulierung. Wir können es auch auf diesem Gebiet nicht hinnehmen, dass wir an Schwung verlieren, sobald der Problemdruck nachlässt. 
In der aktuell kritischen Situation sind die Herausforderungen in Europa besonders drängend. Übermäßige öffentliche Ausgaben haben in einigen Ländern der Eurozone zu untragbar hohen Schuldenständen und Budgetdefiziten geführt, die den wirtschaftlichen Wohlstand gefährden. Um das Vertrauen der Märkte und der Bürger wiederherzustellen, müssen die Regierungen der Eurozone ihre Entschlossenheit zur Konsolidierung der öffentlichen Haushalte aber auch zur Stärkung ihrer volkswirtschaftlichen Wettbewerbsfähigkeit überzeugend demonstrieren.

Mit unserer Unterstützung für Griechenland und später für Irland und Portugal haben wir die Gefahr von Ansteckungseffekten auf andere Länder der Eurozone abgewehrt und damit einen drohenden Zusammenbruch der europäischen und internationalen Finanzmärkte verhindert. Das war ein großer Erfolg, wenngleich nur ein Teilerfolg. Die Lage einiger Mitgliedstaaten der Eurozone, insbesondere Griechenlands, ist weiterhin ernst und verlangt ganz aktuell eine durchgreifende Lösung. Das heißt aber nicht, dass unsere damaligen Akutmaßnahmen falsch waren. Sie sind Teil des Gesamtkonzepts der europäischen Staats- und Regierungschefs mit dem Ziel, die Probleme einiger Mitgliedstaaten in den Griff zu bekommen, um die Eurozone insgesamt auf Dauer zu stabilisieren. Mitgliedstaaten mit hohen Schuldenständen und Defiziten müssen ihre Ausgaben drastisch kürzen, die Einnahmen erhöhen und strukturelle Hindernisse für nachhaltiges Wachstum beseitigen. Mit dieser Strategie ermöglichen wir es den betroffenen Ländern, sich wirtschaftlich zu erholen und erhalten somit die Grundlage für Wachstum und Wohlstand in der gesamten Eurozone. Damit diese Reformen greifen können, brauchen die betroffenen Länder jedoch ein Minimum an Zeit Zeit, die ihnen die Finanzmärkte nicht mehr gewährt hätten. Deswegen mussten wir einspringen, im wohlbedachten Abwägen unseres eigenen, fundamentalen Interesses am Erhalt der Gesamtkonstruktion.

Kritiker unserer Strategie sind der Meinung, dass Haushaltskonsolidierung, Verschlankung des öffentlichen Sektors und flexiblere Arbeitsmärkte einen Wachstumseinbruch in den betroffenen Ländern erst hervorrufen. Selbst wenn dies so wäre, muss man den Ausgleich zwischen kurzfristigen Einschnitten und langfristigem Nutzen suchen. Das dadurch gewonnene Vertrauen bei Verbrauchern und Investoren und der wiederum hierdurch nur mögliche Abbau der Arbeitslosigkeit werden den kurzfristigen Konsumeinbruch auch wieder ausgleichen. Wir müssen gerade jetzt langfristig denken. Allzu lange haben wir den kurzfristigen vor den langfristigen Nutzen gestellt, mit dem Ergebnis, dass Haushalts- und Finanzpolitik immer wieder und immer weiter aus dem Ruder liefen 
und Verschuldung wie Defizite in vielen Fällen ihr überhöhtes Niveau erreicht haben.

Ungünstige wirtschaftliche Fundamentaldaten waren aber nicht der einzige Grund für die dramatische Verschlechterung der Refinanzierungsbedingungen in einigen Teilen der Eurozone. Vielmehr haben die Ereignisse des vergangenen Jahres einmal mehr gezeigt, dass moderne Finanzmärkte die Volatilitäten maßlos erhöhen können. Vor diesem Hintergrund steht die Politik in der Pflicht, die Tendenz der Finanzmärkte zu konterkarieren, die Krise auch noch zu verschärfen. Dies bedeutet aber keineswegs, dass wir auf den Marktzinsmechanismus als Disziplinierungsinstrument verzichten oder ihn außer Kraft setzen wollten. Ganz im Gegenteil: Regierungen sind auf die Märkte angewiesen, weil nur sie Fehlentwicklungen offenbaren, die Regierungen nicht immer hören möchten, und nur sie die Disziplin erzwingen, hieraus die richtigen Konsequenzen ziehen - allerdings oftmals sehr spät und sehr plötzlich.

Selbstverständlich kann es - und dies nicht nur aus wirtschaftlichen Gründen nicht allein die Aufgabe der Steuerzahler sein, die Krisenländer auf ihrem Weg zurück zum Wachstumskurs zu unterstützen. Die Frage, wie wir die Lasten durch die finanziellen Hilfen auf die Finanzinstitutionen einerseits und die Steuerzahler andererseits aufteilen, wird über Jahre hinweg die Stabilität der Finanzmärkte und die Legitimität unserer westlichen Demokratien bei ihren Bürgern entscheidend beeinflussen. Aus diesem Grund stellt eine angemessene Beteiligung des Privatsektors einen integralen Bestandteil unserer Bemühungen dar, den Ländern bei ihren Wirtschaftsreformen zu helfen. Zudem müssen wir den entscheidenden Grundsatz stärken, auf dem eine Marktwirtschaft beruht, um ordnungsgemäß funktionieren zu können - die Untrennbarkeit von Risiko und Haftung. Ohne einen wesentlichen Beitrag der Finanzinstitute wird die Legitimation unseres westlichen Modells Schaden nehmen, nicht nur die Legitimation dieser Finanzinstitute selber.

Gleichzeitig müssen wir gewisse Strukturfehler in der Europäischen Währungsunion korrigieren. Mit der Krise ist der Zeitpunkt gekommen, um die Entwicklung unserer politischen und institutionellen Strukturen auf der europäischen Ebene hin zu einer fiskalischen und politischen Union zu beschleunigen. Andernfalls wird es äußerst schwierig, die Märkte von der langfristigen Tragfähigkeit der Eurozone zu überzeugen. Dies bedeutet vor allem, dass die nationalen Finanz- und Wirtschaftspolitiken der Eurozone besser und verbindlich aufeinander und auf eine gemeinsame Stabilitätsorientierung hin abgestimmt werden müssen. Die Währungsunion braucht also institutionelle Strukturen, die es er- 
möglichen, Mitgliedstaaten der Eurozone zu einer Fiskal- und Haushaltspolitik zu verpflichten, die ihrer Verantwortung für die gemeinsame Währung auch wirklich gerecht wird. Die Europäische Wirtschafts- und Währungsunion wird letztlich nicht erfolgreich sein, wenn einige Länder dauerhaft Defizite aufweisen und ihre Wettbewerbsfähigkeit auf Kosten der Stabilität des Euro insgesamt schwächen, ohne dass dies von europäischer Ebene aus verhindert werden könnte. Um dies zu erreichen, bedarf es einer Revision der vertraglichen Grundlagen 